\title{
Implicit alcohol-related expectancies and the effect of context
}

Monk, R. L. ${ }^{1}$, Pennington, C. R. ${ }^{1}$, Campbell, C. ${ }^{2}$, Price, A. ${ }^{3}$, \& Heim, D. ${ }^{1}$

${ }^{1}$ Department of Psychology, Edge Hill University, Ormskirk, UK

${ }^{2}$ Department of Psychology, University of Ulster, Ulster, UK

${ }^{3}$ School of Health Sciences, Salford University, Salford, UK

Corresponding Author: Rebecca Monk, Department of Psychology,

Edge Hill University, St Helens Road, Ormskirk, Lancashire, L39 4QP

Email: monkre@edgehill.ac.uk

\section{Qualifications}

Rebecca L. Monk: BSc (Hons) Psychology, MSc Forensic Psychology, University of Central Lancashire, PhD Edge Hill University.

Charlotte R. Pennington: BSc (Hons) Psychology, PhD Candidate, Edge Hill University. Email: penninc@edgehill.ac.uk

Claire Campbell: BA (Hons) Psychology, PhD National University of Ireland, Maynooth. Email: c.campbell2@ulster.ac.uk

Alan Price: BSc (Hons) Psychology, Salford University. Email: a.d.price1@edu.salford.ac.uk

Derek Heim: BSc (Hons) Psychology and Politics, PhD University of Strathclyde.

Email: heimd@edgehill.ac.uk

Number of figures: 2

Disclosures

and

Acknowledgements

The authors would like to thank Maddison Barnes, Keelan Donohue and Lydia Suffling of Edge Hill University and Emma-Louise Cully of University of Ulster for their hard work and assistance with data collection.

The authors declare no conflicts of interest. 


\title{
Implicit alcohol-related expectancies and the effect of context
}

\begin{abstract}
Objective: The current study examined the impact of varying pictorial cues and testing contexts on implicit alcohol-related expectancies. Method: Seventy-six participants were assigned randomly to complete an Implicit Relational Assessment Procedure (IRAP) in either a pub or lecture context. The IRAP exposed participants to pictorial cues that depicted an alcoholic beverage in the foreground of a pub (alcoholcongruent stimuli) or university lecture theater (alcohol-incongruent stimuli), and participants were required to match both positive and negative alcohol-related outcome expectancies to these stimuli. Corresponding to a $4 \times 2$ design, IRAP trial-types were included in the analysis as repeated measure variables whereas testing environment was input as a between-participants variable. Results: Participants more readily endorsed that drinking alcohol was related to positive expectancies when responding to alcohol-congruent stimuli and this was strengthened when participants completed the task in a pub. Moreover, they more readily confirmed that alcohol was related to negative expectancies when responding to alcohol incongruent stimuli. Conclusions: These findings suggest that alcohol-related cues and environmental contexts may be a significant driver of positive alcoholrelated cognitions, which may have implications for the design of interventions. They emphasize further the importance of examining implicit cognitions in ecologically valid testing contexts.
\end{abstract}


Key words: Alcohol, Outcome expectancies, Context, Implicit cognitions 


\section{Implicit alcohol-related expectancies and the effect of context}

It is well established that alcohol-related cognitions are an important determinant of consumption (Jones et al., 2001; Pieters et al., 2010). In particular, the anticipated positive outcomes of drinking alcohol have been found to be a significant predictor of alcohol use onset and consumption (e.g., Anderson et al.,, 2011; Christiansen et al., 1989; Lewis \& O'Neill, 2000), whilst negative outcome expectancies have been associated with lower levels of alcohol consumption (e.g., Leigh \& Stacy, 1993, although see Jones, et al., 2001 for a debate). Early research has indicated that placing participants in bar environments, relative to neutral contexts, increased both positive (Wall et al., 2000; 2001) and negative alcohol-related outcome expectancies (Wiers et al., 2003). Positive alcohol expectancies for sexual enhancement have also been shown to be more strongly endorsed in college social settings (LaBrie et al., 2011). More recently, research has expanded the examination of such cognitions, both outside the laboratory (Monk \& Heim, 2013a; 2014; 2015) and under contextual cueing conditions (Monk \& Heim, 2013b). Here it has been revealed that positive outcome expectancies are stronger during exposure to alcohol-related stimuli and when testing occurs in social groups, when contrasted with testing in solitary conditions and under neutral cue exposure (Monk \& Heim, 2013b). These findings suggest that outcome expectancies are not static. Rather, they appear to shift as a function of people's current emotional state (Birch et al., 2004) and environmental and social contexts (Monk \& Heim, 2014)

Whilst providing valuable insights into the context-dependent nature of such cognitions, a shortcoming of this work is its reliance on explicit measures of alcohol expectancies. Explicit outcome expectancies reflect one's self-reported beliefs about the perceived 
likely consequences of drinking. However, as people are aware of such cognitions, these - as with all explicit substance use measures - may be prone to socially desirable responding (Davies, 1997). Conversely, implicit beliefs are inherently less vulnerable to demand characteristics as they occur outside of conscious awareness. Moreover, there has been the suggestion that self-reported (explicit) attitudes do not necessarily reflect internal (implicit) beliefs (Cullen et al., 2009; Power et al., 2009). This notion is supported by research which indicates that explicit and implicit cognitions explain unique variance in alcohol consumption (Pieters et al., 2010; Reich et al., 2010; Rooke et al., 2008; Wiers et al., 2002). As such, implicit and explicit alcohol expectancies may reflect intrinsically different processes (Stanley et al., 2008) and the failure to examine the effect of context on implicit expectancies may represent a limitation in the current literature in this area.

In light of this, it may be pertinent to examine the effect of context on nonconscious (implicit) beliefs about the likely outcomes of drinking alcohol, in other words one's implicit alcohol-related expectancies. Indeed, research methods designed to assess automatic processes indicate that contextual factors may exert an influence on implicit alcohol-related responses. For example, participants show elevated cue reactivity (Petit et al., 2012) and quicker response times (Kreusch et al., 2013) to alcohol-related stimuli using the Go/No-Go Association Task (GNAT). Similarly, research using the Implicit Association Test has shown that heavy drinkers more strongly associate alcohol-related stimuli with positive arousal expectancies compared to light drinkers (c.f. Wiers et al., 2002). Furthermore, such effects have been shown 
to transfer between clinical (Field et al., 2014) and non-clinical groups (Albery et al., 2015), and therefore have implications for intervention. These results suggest that exposure to alcohol-related contexts, where such cues would be expected to be particularly pertinent, may exert a powerful effect on cognitions. Nevertheless, the aforementioned procedures utilized to examine implicit alcohol-related cognitions do not require participants to report the extent to which they hold a particular belief or attitude. Instead, this belief is inferred indirectly by associative responding (c.f., Barnes-Holmes et al., 2011). This means that any variability in the strength to which such beliefs are endorsed across contexts cannot be inferred from such research - a subtle distinction but one which allows for the possibility that certain beliefs may be ubiquitous but their salience may differ depending on one's current situation. The Implicit Relational Assessment Procedure (Barnes-Holmes et al., 2006) was developed to overcome this issue, and measures specific relations among sets of stimuli by asking participants to respond directly to target statements and pictorial cues concerning their actual attitudes or beliefs (Power et al., 2009). As such, the IRAP provides a direct measure of implicit beliefs, requiring participants to respond to these pictorial targets in ways that are either consistent or inconsistent with their established verbal relations (Barnes-Holmes et al., 2010). Recent research has documented the clinical utility of the IRAP, suggesting that implicit outcome expectancies may be predictive of drug abstinence and treatment outcomes (Carpenter et al., 2012).

The current research builds upon previous work to examine whether varying pictorial cues and environmental contexts impact explicit alcohol-related expectancies. By employing the IRAP (Barnes-Holmes et al., 2006), the current research was able to examine implicit responses to both alcohol-related expectancy 
words and visual stimuli. Here, contextually congruent or non-contextually congruent pictorial cues were placed alongside positive and negative outcome expectancy phrases. It was predicted that participants would more readily endorse positive expectancies when responding to alcohol-congruent stimuli (a picture of a pint in a pub), but endorse negative expectancies when viewing alcohol incongruent stimuli (a picture of a pint in a lecture theatre). In other words, response times were expected to be shorter for the "alcohol-congruent good" trial type (in contrast to the alcoholincongruent stimuli good, alcohol-congruent stimuli bad and alcohol-incongruent stimuli bad trial types). Conversely, response times were expected to be longer for the "alcohol-incongruent bad" trial type (in contrast the other three trial types). The IRAP was also administered in two real-life testing contexts, namely a pub and lecture theatre. Previous research has demonstrated that there is an interactive effect of testing context on explicit expectancy measures, such that, both the type of visual stimuli (alcohol vs neutral) and one's current social context (solitary vs group testing) impact cognition. For example, increases in explicit positive expectations have been shown to be the result of being among peers and under the influence of alcoholrelated cues, whist peer effects were not observed in the absence of pub based stimuli (Monk \& Heim, 2013). Accordingly, such interaction effects were anticipated in implicit measures, and it was hence predicted that response times to positive expectancy items would be quicker during in-vivo administration of the IRAP in an alcohol-related environment (Pub) as opposed to a neutral, non-alcohol-related context (Lecture theatre). Such findings would extend previous research to further suggest that real life alcohol-related contexts and visual stimuli have an effect on implicit alcohol-related expectancies.

\section{Method}




\section{Participants}

Seventy-six undergraduate students (Mage $=18.91 S D=.70,45 \%$ male $)$ who were self-reported drinkers were recruited via responses to online advertisements. They were assigned randomly to either the pub testing context $(n=40)$ in which participants completed the experiment in the University Pub, or the lecture context ( $n$ =36). Fourteen additional participants were originally tested but were subsequently excluded when they did not meet the IRAP performance criteria (see results section). Average AUDIT scores for this sample were $13.2(S D=5.52)$, above the cut-off for clinical assessment (scores of 8 or above are deemed to indicate hazardous or harmful alcohol use - Babor et al., 2001), which is comparable with recent research also using a UK student sample (Clarke et al., 2015; Moss et al., 2015).

\section{Materials}

\section{Self-Report Measures}

Alcohol Use Disorders Identification Test (AUDIT). The AUDIT (Saunders et al., 1993) comprises 10 self-report items that measure the frequency and quantity of alcohol consumption, drinking behavior and alcohol-related problems. Responses to statements such as "How often during the last year have you found that you were not able to stop drinking once you had started" are recorded on a 5-point Likert scale $(0=$ Never, $4=4$ or more times). Internal consistency was high on this measure $($ Cronbach's Alpha $=.82)$.

\section{Alcohol Outcome Expectancy. The Alcohol Outcome Expectancy} questionnaire (Leigh \& Stacy, 1993) outlines 32 potential positive and negative outcomes of alcohol consumption, such as "I feel happy" or "I have problems with memory and concentration". Participants are asked to rate the likelihood of each 
outcome on a 6-point Likert scale ( $1=$ No chance, 6 = Certain to happen). Responses to positive and negative outcome expectancy items showed high internal consistency (Cronbach's $a=.92 \& .84$, respectively).

\section{Design and Procedure}

A 4 (Trial-type; Alcohol-congruent stimuli good, alcohol-incongruent stimuli good, alcohol-congruent stimuli bad, and alcohol-incongruent stimuli bad) x 2 (Testing Context; Pub, lecture) mixed-design was utilized within this research. Trial-type was included as a repeated measure variable whereas testing environment acted as a between-participants variable. Participants were recruited via an online participation pool, and were allocated randomly to complete all aspects of the experiment in either the lecture theatre or pub testing conditions ${ }^{1}$. Specifically, once allocated to a condition, participants were sent an email to make arrangements to meet the researchers at the appropriate testing context. Due care was taken to ensure that the contexts were similar in terms of environmental distraction and noise. To this end, testing took place between $12 \mathrm{pm}$ and $6 \mathrm{pm}$ to limit noise and participants were seated at a quiet area in the pub to complete the tasks. The researcher ensured that participants were familiar with the task requirements before they completed between two and eight practice blocks of IRAP trials (dependent on proficiency, which was defined as response accuracy $\geq 70 \%$ and median response latencies $\leq 2,000 \mathrm{~ms}$ ). Practice blocks contained modified variations of the stimuli used in the experimental trials, in order to control for any effect of variable exposure to experimental stimuli across participants. They then completed six critical test blocks of the IRAP procedure. Upon completion of the IRAP, participants completed a series of demographic questions and the AUDIT and alcohol expectancy questionnaires, in 
order to ensure that there were no between-participant differences as a function of the testing context. These questionnaires remained the final components in order to limit the signal strength of the study - whereby participants may infer the aims of the research from the experimental methodology and thus alter their behavior in order to fulfil the perceived demands of the study (c.f., Davies \& Best, 1996).

The IRAP (Barnes-Holmes et al., 2006) is an automated, latency-based task that works on the premise that it should be quicker to map two concepts onto a single response option when those two concepts are related in memory. Participants were required to respond quickly and accurately to a series of trials in order to assess the anticipated positive and negative consequences of alcohol as a function of situational context (i.e., pub or lecture) and pictorial cue (alcohol-incongruent stimuli or alcoholcongruent stimuli). Each trial presented one of two pictorial target stimuli that portrayed an alcohol beverage (i.e., a beer bottle) in the foreground of a pub (alcoholcongruent stimuli), or a university lecture theatre (alcohol-incongruent stimuli). Below the target, in the middle of the screen, one of 12 target phrases were presented randomly. These target phrases were adapted from previous research on drug-related expectancies (Carpenter et al., 2012) and contained 6 positive expectancy phrases, (i.e., I am sexier, I am in control, I am friendlier, I am nicer, I am talkative and I am outgoing) and 6 negative outcome expectancies (i.e., I am jumpy, I am aggressive, I am alone, I am tense, I am mean, I want to fight). Accordingly, during each block of trials participants were exposed to a random assortment of four distinct trail-types: Alcohol-congruent stimuli paired with positive expectancy phrases, alcohol-congruent stimuli paired with negative expectancy phrases, alcohol-incongruent stimuli paired with positive expectancy phrases, and alcohol-incongruent stimuli paired with negative expectancy phrases (See Figure 1). There were 24 trials in each block (6 
exposures to each of the four trial-types) and a total of 8 blocks (excluding practice blocks).

\section{[INSERT FIGURE 1 HERE]}

Participants were asked to respond using two response options, 'Likely' and 'Unlikely' which were presented at the bottom left and right of a laptop computer screen. Positioning of these two response options switched from left to right in a quasi-random order to ensure that they could not appear in the same left-right position more than three times in succession. Participants were required to respond to each trial in a way that was is either consistent or inconsistent with their pre-experimentally determined verbal relations. During consistent trials, participants were instructed that they should match positive valence terms (e.g., I am friendlier) to alcohol congruent stimuli (pub) and match negative valence terms (e.g., I am aggressive) to alcohol incongruent stimuli (lecture) by pressing 'Likely'. When the reverse mappings were shown (e.g. alcohol congruent stimuli with a negative term, and alcohol incongruent stimuli with a positive term), they were instructed to press 'Unlikely'. In inconsistent trials, participants were told to adopt the opposite pattern of responding, namely relating negative terms to alcohol-congruent stimuli and positive terms to alcoholincongruent stimuli. The consistent and inconsistent blocks of trials appeared in alternating order and the designation of the block that participants began with was counterbalanced across participants. Each correct response resulted in the removal of all stimuli from the screen for $400 \mathrm{~ms}$ after which the next trial was presented. Participants were notified of an incorrect response with the presentation of a red ' $\mathrm{X}$ ', which remained on the computer screen until the participant selected the correct 
response. The latency for each response was recorded from the onset of the stimulus to the emission of a correct response, and any errors were included in this latency ${ }^{2}$. Participants' response times for each trial-type were used to calculate four $D$-scores, corresponding to each IRAP trial-type. This $D$-score was computed in line with an adaptation of Greenwald et al.'s, (2003, p. 214) improved scoring algorithm (c.f., Campbell et al., 2011), which provides additional statistical power relative to the original scoring procedure and minimizes the impact of factors such as age, motor skills and cognitive ability on latency data (Carpenter et al., 2012). It functions to characterize the difference in participants' response times between consistent $($ alcohol-congruent $=$ likely, alcohol-incongruent $=$ unlikely $)$ and inconsistent trials (alcohol-congruent $=$ unlikely, alcohol-incongruent $=$ likely $).$

\section{Results}

Participants in the pub and lecture testing contexts did not differ significantly in their age, gender, AUDIT scores (including overall score and consumption and negative consequences sub-components) or explicit positive and negative expectancies ( $p>.05$ in all cases). In accordance with recommendations, the 76 included participants met the criteria of above $70 \%$ accuracy or with a median response time of less than 2,000 milliseconds in order to ensure that responses were are accurate and quick (and thus non-conscious/implicit, c.f., Barnes-Holmes et al., 2010; Campbell et al., 2011; Vahey et al., 2009). The excluded participants $(n=14)$ did not differ significantly from the included participants in terms of age, gender, baseline positive or negative expectancies or AUDIT scores $(p>.05)$. A total of four $D$-scores, corresponding to each IRAP trial-type, were calculated in line with an adaptation of Greenwald et al.'s (2003) improved scoring algorithm. The steps involved in calculating these steps were 
as follows: (i), only response latency data from critical test blocks were used; (ii), latencies above 10,000 $\mathrm{ms}$ were eliminated from the data set; (iii) all data for participants were removed if they produced more than $10 \%$ of test blocks with latencies below $300 \mathrm{~ms}$; (iv) for each trial-type, an overall standard deviation for all trails was calculated; (v) two mean latencies were calculated for each trial-type, the overall mean for consistent and inconsistent trials; (vi) the difference scores were calculated by subtracting the mean latency score from the consistent trials (alcoholcongruent $=$ good, alcohol-incongruent $=$ bad) from the mean latency of the inconsistent trials $($ alcohol-congruent $=$ bad, alcohol-incongruent $=$ good); $($ vii $)$ the difference scores were divided by the standard deviation calculated in step (iv), yielding one overall $D$-score for each trial-type. Responding on each of the 4 trialtypes is depicted in Figure 2. For clarity of interpretation, the mean $D$-scores for the trial-type of 'Alcohol incongruent - Good' and 'Alcohol incongruent - Bad' were inverted so that positive scores indicate that participants endorsed positive alcoholrelated expectancies and negative $D$-scores indicate that participants endorsed negative alcohol-related expectancies ${ }^{2}$.

[INSERT FIGURE 2 HERE]

Response latencies were subjected to a 4 (trial-type; alcohol-congruent stimuli good, alcohol-incongruent stimuli good, alcohol-congruent stimuli bad, alcohol-incongruent stimuli bad) $\times 2$ (testing context; pub or lecture) mixed Analysis of Variance (ANOVA). Results indicated a significant main effect of trial-type $^{3}, F(3,222)=10.57, p<.001, \eta_{p}^{2}=.13$, but no significant main effect of context $F(1,74)=.99, p>.05, \eta_{p}^{2}=.01$. These results were qualified by a significant two-way interaction between trial-types and the testing context, $F(3,222)=5.59, p<.05, \eta_{p}^{2}=.07$, which was elucidated using simple main effects analyses (linearly independent pairwise comparisons). Betweencontext comparisons revealed that participants exposed to alcohol-congruent stimuli endorsed positive expectancies more strongly when they were in the pub $(M=.24, S D=.26)$ relative to a lecture context $(M=.08, S D=.39) p<$ 
.05. No other comparisons were significant as a function of testing context, $p$ $>$.05. Between trial-type comparisons were as follows:

\section{Lecture Testing Context}

Contrasts between the alcohol congruent-good trial type and both the alcohol incongruent-good and incongruent-bad trial types were statistically significant (all $p<.05$ ). Participants more readily endorsed positive expectancies for positive-congruent trials $(M=.08, S D=.39)$, relative to positive incongruent $(M=-.07, S D=.39)$. They also endorsed positive expectancies to congruentgood trials $(M=.08, S D=.39)$, but in contrast endorsed negative expectancies for alcohol incongruent-bad trials $(M=-.10, S D=.36)$. No other within-subjects contrasts by trial type were statistically significant, $p>.05$.

\section{Pub Testing Context}

Contrasts between the alcohol-congruent good trial type and alcoholcongruent bad, alcohol-incongruent good, and alcohol-incongruent bad trial types were all statistically significant (all $p<.05$ ). Participants more readily endorsed positive expectancies for positive alcohol-congruent trials $(M=.24$, $S D=.26)$ than for negative congruent $(M=.09, S D=.40)$, positive alcoholincongruent $(M=-.10, S D=.32)$, and negative alcohol-incongruent trials $(M=$

$-.14, S D=.35)$. No other contrasts were significant, within one exception ${ }^{4}$. Sensitivity analyses were conducted in light of concerns raised by participants in respect of one of the questions (see further details in the discussion). These analyses, however, revealed no significant alterations to the current findings when this item was removed ${ }^{5}$. 
Overall, results reveal that participants tested in a lecture context more readily confirmed that positive outcome expectancies were related to alcohol-congruent stimuli relative to negative expectancies, which they associated with alcoholincongruent stimuli. Moreover, this effect was pronounced for participants tested in a pub context who consistently responded that positive expectancies were related to alcohol-congruent stimuli whereas negative expectancies were related to alcohol incongruent stimuli. This suggests that participants' environment had an interactive effect on implicit outcome expectancies.

\section{Discussion}

The purpose of the current study was to examine how varying pictorial cues and reallife testing contexts may impact implicit alcohol-related outcome expectancies. For the first time, the current research demonstrated that alcohol-related pictorial cues and real-time contexts can have an interactive effect on implicit positive and negative expectancies. In accordance with predictions, participants implicitly endorsed that positive expectancies were related to alcohol-congruent stimuli, in comparison to negative expectancies, and this effect was heightened when they were tested in a pub context relative to a lecture theatre. In other words, implicit beliefs about the likely positive outcomes of consumption appear to be higher when exposed to alcoholcongruent stimuli in a pub environment. Moreover, the same participants more readily endorsed that negative outcomes were related to alcohol-incongruent stimuli demonstrating that they were able to discriminate between positive and negative alcohol expectancies as a function of alcohol-congruent and incongruent cues.

Given that the IRAP works by assessing the relational nature and strength of memories and beliefs (Barnes-Holmes et al., 2006), the current findings suggest that being in a pub environment may make the association between alcohol-related stimuli 
and the expectation of positive outcomes more salient. From this perspective, the effects observed in the current study may be analogous to real-life cueing processes occurring in alcohol-related environments. This adds to previous research which suggests that pub testing contexts can heighten explicit outcome expectancies (e.g., Monk \& Heim, 2013a). Specifically, it is suggested that exposure to alcoholcongruent stimuli inside an environment where drinking takes place may prove a particularly powerful influence on alcohol consumption and associated cognitions (Monk \& Heim, 2014; Monk et al., 2015). In particular, the current research suggests that these results are replicated using implicit stimuli, thus allaying previous concerns that demand characteristics and procedural signalling may have explained such findings.

The apparent cumulative effect of pictorial stimuli and testing context was also apparent in IRAP trial-type comparisons. Whilst testing occurred in the lecture context, participants' responses to alcohol-congruent stimuli paired with negative expectancy words (i.e., Alcohol congruent-Bad) did not differ from responses to alcohol-incongruent stimuli paired with either positive or negative expectancy items (i.e., Alcohol incongruent-Good or Bad). In contrast, all of these contrasts were significant in the pub-testing environment. Here, participants more strongly endorsed positive expectancies in relation to alcohol-congruent stimuli relative to negative expectancies. Further, participants in the pub more readily endorsed negative expectancies, relative to positive expectancies in response to alcohol-incongruent stimuli. This suggests that, on their own, alcohol-congruent stimuli may be enough to trigger implicit positive expectancies, whilst alcohol-incongruent images appear to elicit negative expectations. Consequently, experimental instructions which necessitated a response that was contrary to one's triggered beliefs appeared to be 
difficult. This effect, however, only occurred during testing in an alcohol-salient environment. Such findings accord with previous work pointing to changes in implicit responses when presenting participants with alcohol-related experimental stimuli (Kreusch et al., 2013; Petit et al., 2012). Furthermore, the current findings suggest that not only may alcohol-related stimuli impact implicit beliefs, but also the context of this exposure may have a significant impact on the implicit expression of these beliefs. Given that researchers suggest that manipulating expectancies might facilitate a targeted reduction in alcohol consumption (Jones et al., 2001; Wood et al., 2007), the current research offers potential insights which may aid the development of interventions. That is, successful interventions must be able to respond to the contextually varying nature of the factors that impact substance use (Davies, 1997). Therefore the present research suggests that cue exposure (expectancy activation) may be beneficial if it precedes attempts to change implicit expectancies, with the intention of reducing alcohol consumption. Indeed, research by Wiers and colleagues has utilized cognitive bias modification training in order to reduce alcohol craving (2015a) and subsequent consumption (2015b) which can result from alcohol-related cue exposure (c.f., Wiers et al., 2013 for a review on such intervention approaches). The present results may also have important implications for research in terms of emphasizing the importance of ecologically valid testing contexts when assessing implicit cognitions.

There are a number of potential limitations to the current research that must be noted. In order to examine the impact of varying pictorial cues on implicit alcoholrelated expectancies, the IRAP stimuli consisted of an alcoholic beverage presented in the foreground of a pub or a lecture theatre. However, it should be highlighted that this design is not fully matched, in that neutral stimuli (e.g., a bottle of water) was not 
utilized. This decision was taken in order to minimise the number of procedural trials, and thus limit the demand placed on participants' time. However, it must therefore be present research does not offer insights into the positive or negative associations that may be activated as a response to non-alcoholic beverages in alcohol and non-alcoholrelated contexts. In other words, the present results cannot be asserted to be alcohol specific. Future research may therefore be advised to examine the effect of alcoholneutral stimuli on implicit responses, to further elucidate the nature of such implicit cognitions.

Furthermore, whilst sensitivity analyses allayed fears about the particular questions unduly affecting results, there are further issues potentially worthy of note. Although care was taken to try and ensure that the testing contexts were similar (in terms of environmental distraction and noise) it is important to acknowledge that fundamental differences in the nature of these two natural contexts may have resulted in greater participant distraction in the pub context. Whilst such variations are perhaps unavoidable to an extent, future research in a larger array of alcohol-related and neutral testing contexts may provide further assurances as to the veracity and generalizability of the current findings. Finally, it should be acknowledged that the current results may not generalize to a wider, non-student sample. This fact may be particularly evident in the relatively high AUDIT scores observed in this sample. One explanation of such heightened scores is that students are immersed in a drinking culture which encourages consumption (Borsari \& Carey, 2001). If one takes this perspective, it is perhaps unsurprising that the AUDIT scores in the present sample are relatively high. An alterative explanation on these scores is that the participants who volunteered for this study did so because of their heavy drinking and positive alcohol-related cognitions. Such self-selecting sample biases are a perennial issue 
within such research areas. The present AUDIT scores are comparable with those from similar UK student samples (e.g. Clarke et al., 2015; Moss et al., 2015). Nonetheless, caution should be taken when attempting to apply such results to samples with lower AUDIT scores, who may thus possess manifestly different implicit cognitions.

In conclusion, previous research has suggested that the cognitive anticipation of the positive consequences associated with drinking alcohol may be a predictor of consumption (Cumsille et al., 2000). To date, however, research has largely examined explicit alcohol-related expectancies or implicit expectancies without consideration of the potential effects of alcohol-related stimuli and people's environment. Addressing these issues, findings from the current study suggest that exposure to alcoholcongruent stimuli inside an environment where drinking takes place may strengthen implicit alcohol-related expectancies. This has potential implications for interventions that aim to tackle problem drinking by attempting to change the cognitions that compel consumption. Future research may benefit from examining whether implicit outcome expectancies are predictive of alcohol consumption, particularly in environments were alcohol-related cues are salient. Further, implicit measures may present as a useful tool to examine alcohol-related expectancies that are not limited by self-presentational motives. 


\section{References}

Albery, I. P., Sharma, D., Noyce, S., Frings, D., \& Moss, A. C. (2015). Testing a frequency of exposure hypothesis in attentional bias for alcohol-related stimuli amongst social drinkers. Addictive Behaviors Reports, 1, 68-72. doi:10.1016/j.abrep.2015.05.001.

Anderson, K. G., Grunwald, I., Bekman, N., Brown, S. A., \& Grant, A. (2011). To drink or not to drink: motives and expectancies for use and nonuse in adolescence. Addictive Behaviors, 36(10), 972-979. doi:10.1016/j.addbeh.2011.05.009. Medline

Babor, T. F., Higgins-Biddle, J. C., Saunders, J. B., \& Monteiro, M. G. (2001). The Alcohol Use Disorders Identification Test (AUDIT): Guidelines for use in primary care. World Health Organisation.

Barnes-Holmes, D., Barnes-Holmes, Y., Power, P., Hayden, E., Milne, R., \& Stewart, I. (2006). Do you really know what you believe? Developing the Implicit Relational Assessment Procedure (IRAP) as a direct measure of implicit beliefs. The Irish Psychologist, 32, 169-177.

Barnes-Holmes, D., Barnes-Holmes, Y., \& Stewart, I. (2010). A sketch of the implicit relational assessment procedure (IRAP) and the relational elaboration and coherence (REC) model. The Psychological Record, 60, 527-542.

Barnes-Holmes, D., Murphy, A., \& Barnes-Holmes, Y. (2010). The implicit relational assessment procedure: Exploring the impact of private versus public contexts and the response latency criterion on pro-white and anti-black stereotyping among white Irish individuals. The Psychological Record, 60, 57-66.

Birch, C. D., Stewart, S. H., Wall, A. M., McKee, S. A., Eisnor, S. J., \& Theakston, J. A. (2004). Mood-induced increases in alcohol expectancy strength in internally motivated drinkers. Psychology of Addictive Behaviors, 18(3), 231-238. doi:10.1037/0893-164X.18.3.231. Medline

Borsari, B., \& Carey, K. B. (2001). Peer influences on college drinking: a review of the research. Journal of Substance Abuse, 13(4), 391-424. doi:10.1016/S08993289(01)00098-0. Medline

Campbell, C., Barnes-Holmes, Y., Barnes-Holmes, D., \& Stewart, I. (2011). Exploring screen presentations in the implicit relational assessment procedure (IRAP). International Journal of Psychology \& Psychological Therapy, 11, 377388.

Carpenter, K. M., Martinez, D., Vadhan, N. P., Barnes-Holmes, D., \& Nunes, E. V. (2012). Measures of attentional bias and relational responding are associated with behavioral treatment outcome for cocaine dependence. The American Journal of Drug and Alcohol Abuse, 38(2), 146-154. doi:10.3109/00952990.2011.643986. Medline

Christiansen, B. A., Smith, G. T., Roehling, P. V., \& Goldman, M. S. (1989). Using alcohol expectancies to predict adolescent drinking behavior after one year. Journal of Consulting and Clinical Psychology, 57(1), 93-99. doi:10.1037/0022006X.57.1.93. Medline 
Clarke, N. C., Field, M., \& Rose, A. K. (2015). Evaluation of a brief personalised intervention for alcohol consumption in college students. PLoS ONE, 10(6), e0131229. doi:10.1371/journal.pone.0131229. Medline

Cullen, C., Barnes-Holmes, D., Barnes-Holmes, Y., \& Stewart, I. (2009). The Implicit Relational Assessment Procedure (IRAP) and the malleability of ageist attitudes. The Psychological Record, 59, 591-620.

Cumsille, P. E., Sayer, A. G., \& Graham, J. W. (2000). Perceived exposure to peer and adult drinking as predictors of growth in positive alcohol expectancies during adolescence. Journal of Consulting and Clinical Psychology, 68(3), 531-536. doi:10.1037/0022-006X.68.3.531. Medline

Davies, J. B. (1997). The Myth of Addiction. ( $2^{\text {nd }}$ edition), Amsterdam: Harwood Academic Publishers.

Davies, J. B., \& Best, D. W. (1996). Demand characteristics and research into drug use. Psychology \& Health, 11(2), 291-299. doi:10.1080/08870449608400258.

Field, M., Marhe, R., \& Franken, I. H. (2014). The clinical relevance of attentional bias in substance use disorders. CNS Spectrums, 19(3), 225-230. doi:10.1017/S1092852913000321. Medline

Greenwald, A. G., Nosek, B. A., \& Banaji, M. R. (2003). Understanding and using the implicit association test: I. An improved scoring algorithm. Journal of Personality and Social Psychology, 85(2), 197-216. doi:10.1037/0022-3514.85.2.197. Medline

Jones, B. T., Corbin, W., \& Fromme, K. (2001). A review of expectancy theory and alcohol consumption. Addiction (Abingdon, England), 96(1), 57-72. doi:10.1046/j.1360-0443.2001.961575.x. Medline

Kreusch, F., Vilenne, A., \& Quertemont, E. (2013). Response inhibition toward alcohol-related cues using an alcohol go/no-go task in problem and non-problem drinkers. Addictive Behaviors, 38(10), 2520-2528. doi:10.1016/j.addbeh.2013.04.007. Medline

Leigh, B. C., \& Stacy, A. W. (1993). Alcohol outcome expectancies: Scale construction and predictive utility in higher order confirmatory models. Psychological Assessment, 5(2), 216-229. doi:10.1037/1040-3590.5.2.216.

Lewis, B. A., \& O’Neill, H. K. (2000). Alcohol expectancies and social deficits relating to problem drinking among college students. Addictive Behaviors, 25(2), 295-299. doi:10.1016/S0306-4603(99)00063-5. Medline

Monk, R. L., \& Heim, D. (2013a). Environmental context effects on alcohol-related outcome expectancies, efficacy, and norms: a field study. Psychology of Addictive Behaviors, 27(3), 814-818. doi:10.1037/a0033948. Medline

Monk, R. L., \& Heim, D. (2013b). Panoramic projection: affording a wider view on contextual influences on alcohol-related cognitions. Experimental and Clinical Psychopharmacology, 21(1), 1-7. doi:10.1037/a0030772. Medline

Monk, R. L., \& Heim, D. (2014). A real-time examination of context effects on alcohol cognitions. Alcoholism, Clinical and Experimental Research, 38(9), 24542459. doi:10.1111/acer.12504. Medline 
Monk, R. L., Heim, D., Qureshi, A., \& Price, A. (2015). "I have no clue what I drunk last night" using Smartphone technology to compare in-vivo and retrospective self-reports of alcohol consumption. PLoS ONE, 10(5), e0126209. doi:10.1371/journal.pone.0126209. Medline

Moss, A. C., Spada, M. M., Harkin, J., Albery, I. P., Rycroft, N., \& Nikčević, A. V. (2015). 'Neknomination': Predictors in a sample of UK university students. Addictive Behaviors Reports, 1, 73-75. doi:10.1016/j.abrep.2015.05.003.

Petit, G., Kornreich, C., Noël, X., Verbanck, P., \& Campanella, S. (2012). Alcoholrelated context modulates performance of social drinkers in a visual Go/No-Go task: a preliminary assessment of event-related potentials. PLoS ONE, 7(5), e37466. doi:10.1371/journal.pone.0037466. Medline

Pieters, S., van der Vorst, H., Engels, R. C. M. E., \& Wiers, R. W. (2010). Implicit and explicit cognitions related to alcohol use in children. Addictive Behaviors, 35(5), 471-478. doi:10.1016/j.addbeh.2009.12.022. Medline

Power, P., Barnes-Holmes, D., Barnes-Holmes, Y., \& Stewart, I. (2009). The Implicit Relational Assessment Procedure (IRAP) as a measure of implicit relative preferences: A first study. The Psychological Record, 59, 621-640.

Reich, R. R., Below, M. C., \& Goldman, M. S. (2010). Explicit and implicit measures of expectancy and related alcohol cognitions: a meta-analytic comparison. Psychology of Addictive Behaviors, 24(1), 13-25. doi:10.1037/a0016556. Medline

Rooke, S. E., Hine, D. W., \& Thorsteinsson, E. B. (2008). Implicit cognition and substance use: a meta-analysis. Addictive Behaviors, 33(10), 1314-1328. doi:10.1016/j.addbeh.2008.06.009. Medline

Saunders, J. B., Aasland, O. G., Babor, T. F., de la Fuente, J. R., \& Grant, M. (1993). Development of the alcohol use disorders identification test (AUDIT): WHO collaborative project on early detection of persons with harmful alcohol consumption--II. Addiction (Abingdon, England), 88(6), 791-804. doi:10.1111/j.1360-0443.1993.tb02093.x. Medline

Senchak, M., Leonard, K. E., \& Greene, B. W. (1998). Alcohol use among college students as a function of their typical social drinking context. Psychology of Addictive Behaviors, 12(1), 62-70. doi:10.1037/0893-164X.12.1.62.

Stanley, D., Phelps, E., \& Banaji, M. (2008). The neural basis of implicit attitudes. Current Directions in Psychological Science, 17(2), 164-170. doi:10.1111/j.14678721.2008.00568.x.

Vahey, N. A., Barnes-Holmes, D., Barnes-Holmes, Y., \& Stewart, I. (2009). A first test of the implicit relational assessment procedure (IRAP) as a measure of selfesteem: Irish prisoner groups and university students. The Psychological Record, 59, 371-388.

Wall, A. M., McKee, S. A., Hinson, R. E., \& Goldstein, A. (2001). Examining alcohol outcome expectancies in laboratory and naturalistic bar settings: a within-subject experimental analysis. Psychology of Addictive Behaviors, 15(3), 219-226. doi:10.1037/0893-164X.15.3.219. Medline

Wall, A. M., McKee, S. A., \& Hinson, R. E. (2000). Assessing variation in alcohol outcome expectancies across environmental context: an examination of the 
situational-specificity hypothesis. Psychology of Addictive Behaviors, 14(4), 367375. doi:10.1037/0893-164X.14.4.367. Medline

Wiers, R. W., van Woerden, N., Smulders, F. T., \& de Jong, P. J. (2002). Implicit and explicit alcohol-related cognitions in heavy and light drinkers. Journal of Abnormal Psychology, 111(4), 648-658. doi:10.1037/0021-843X.111.4.648. Medline

Wiers, R. W., Wood, M. D., Darkes, J., Corbin, W. R., Jones, B. T., \& Sher, K. J. (2003). Changing expectancies: cognitive mechanisms and context effects. Alcoholism, Clinical and Experimental Research, 27(2), 186-197. doi:10.1097/01.ALC.0000051023.28893.8A. Medline

Wiers, R. W., Gladwin, T. E., Hofmann, W., Salemink, E., \& Ridderinkhof, K. R. (2013). Cognitive bias modification and cognitive control training in addiction and related psychopathology mechanisms, clinical perspectives, and ways forward. Clinical Psychological Science, 216(2), 192-212. doi:10.1177/2167702612466547.

Wiers, C. E., Stelzel, C., Gladwin, T. E., Park, S. Q., Pawelczack, S., Gawron, C. K., et al. (2015a). Effects of cognitive bias modification training on neural alcohol cue reactivity in alcohol dependence. The American Journal of Psychiatry, 172(4), 335-343. doi:10.1176/appi.ajp.2014.13111495. Medline

Wiers, R. W., Houben, K., Fadardi, J. S., van Beek, P., Rhemtulla, M., \& Cox, W. M. (2015b). Alcohol cognitive bias modification training for problem drinkers over the web. Addictive Behaviors, 40, 21-26. doi:10.1016/j.addbeh.2014.08.010. Medline

Wood, M. D., Capone, C., Laforge, R., Erickson, D. J., \& Brand, N. H. (2007). Brief motivational intervention and alcohol expectancy challenge with heavy drinking college students: a randomized factorial study. Addictive Behaviors, 32(11), 2509-2528. doi:10.1016/j.addbeh.2007.06.018. Medline 

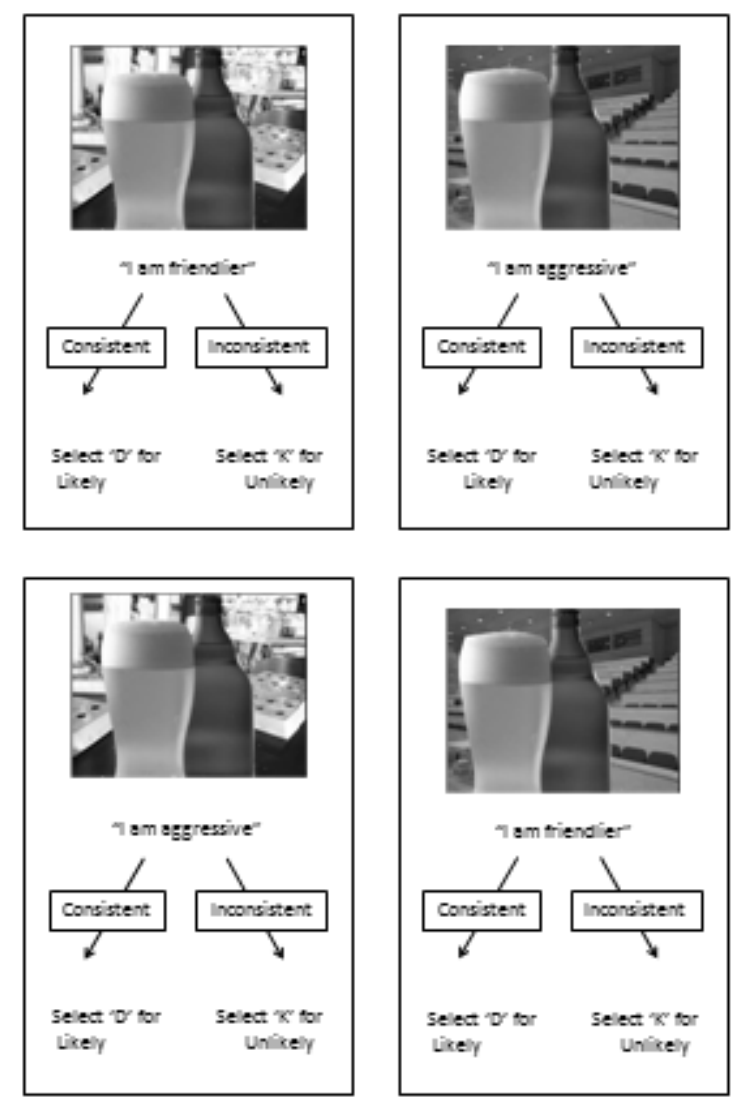

Figure 1. Example of each IRAP trial-type. 


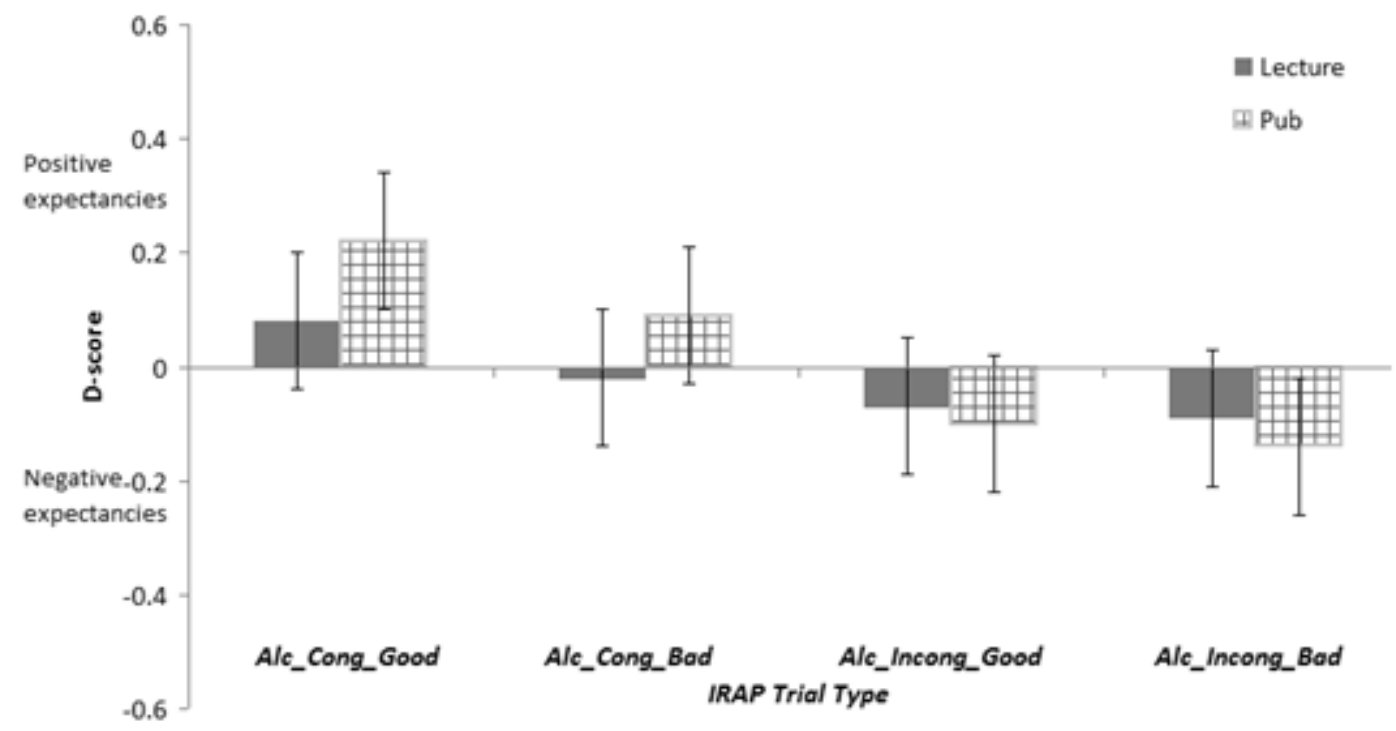

N.B Positive scores indicate that participants endorsed positive alcohol-related expectancies and negative $D$-scores indicate that participants endorsed negative alcohol-related expectancies

Figure 2. The mean $D$-scores for each trial-type as a function of testing context (error

bars represent standard error)

${ }^{1}$ Pilot research for this study utilized opportunity sampling but it was decided that random allocation would be beneficial in order to allay fears that those who were recruited in the pub testing condition could have been manifestly different from those who were tested in the lecture condition. Such concerns arise from suggestions that people seek out supportive social environments for their drinking and, as such, those who drink more (and have supportive cognitions) may be more likely to frequent a student pub (Senchak, Leonard, and Greene, 1998).

${ }^{2}$ In Greenwald et al.'s (2003) improved scoring algorithm, latency errors are analyzed within the final data as they enhance IAT effects. Specifically, as errors are more frequently associated with inconsistent trials, the inclusion of these errors results in slower responding for these trials and therefore increases the accuracy of D-scores.

${ }^{3}$ Pairwise comparisons indicated a significant difference between the trial-types alcohol-congruent positive expectancy words and alcohol-incongruent positive expectancy words. Here participants affirmed that alcohol-congruent stimuli was related to positive drinking expectancies $(M=.16, S D$ $=.33)$ but that alcohol-incongruent stimuli was related to negative drinking expectancies $(M=-.09, S D$ $=.36), p<.001$. No other trial-type comparisons reached significance, $p>.05$.

${ }^{4}$ (Note, the contrast between alcohol incongruent-good and negative alcohol-congruent trials were also significant, but is not reported here as it do not relate to the set hypotheses).

${ }^{5}$ Some participants reported that they found it difficult to match the expectancy item "I am alone" to the lecture pictorial stimuli as this contrasted with the expectancy that they were typically with a large group in this environment. This supports the ideation that participants respond in line with their consistent and inconsistent verbal relations (Barnes-Holmes et al., 2006). However, it is conceivable that removing this particular item may heighten the effect sizes obtained in the current research. Sensitivity analyses (removing this item) also replicated the effects found here, thus allaying fears that this item may have unduly affected results. 